\title{
Study on the Impact of IT Infrastructure Capability on Cooperative Inter-Firm Relationship
}

\author{
GUO Jing \\ School of Management \\ Xi'an Polytechnic University \\ Xi'an, China \\ 396509318@qq.com
}

\begin{abstract}
This study seeks to assess the impact of IT infrastructure capability on cooperative inter-firm relationship, called electronic cooperation. Grounded in IT infrastructure framework and transaction cost and information processing theories, we identify multiple dimensions of IT infrastructure capability- IT resources, information resources, and process resources and propose a model and formulate hypotheses, which articulate relationships between the dimensions and electronic cooperation. We then test the hypotheses empirically using the data from a survey of IS managers in manufacturing and retailing industries. The results support the multi-dimensional view of IT infrastructure capability in inter-firm relationship.
\end{abstract}

Keywords-IT infrastructure capability;Interfirm relationship; Electronic cooperation; IT resources

\section{INTRODUCTION}

Firms have used a variety of information technologies (IT) to do businesses with their partners within supply chains, which include Internet, electronic data interchange (EDI), interorganizational information systems (IOS), and/or combination of them. There are increasing numbers of studies that view such information technologies as inter-organizational IT infrastructure (e.g., [5, 8]). It has been viewed as major means to coordinate and communicate to partnering firms and to support B2B commerce [1, 4]. In fact, firm shave been used such IT infrastructure to manage inventory via electronic links to suppliers, to strengthen business process integration with business partners, to support inter-firm business process redesign [12], and to design and customize products and services with customers and suppliers[9].

Such IT infrastructure alters efficiency, business processes, and power/domination relationships within supply chains and it has been said to enable firms to build a new form of relationships in their value chains[4, 13].For instance, firms within a competitive industry may use IT infrastructure to unite their business processes under common set of standards and protocols, and the standards help firms to establish entry or exit barriers and create a more oligopolistic market structure. IT infrastructure has been used to manage coordination processes between firms and to make the workflow systems in relationship be tightly integrated. In addition, the increasing transparency offered by
Internet-based information systems often forces companies to move toward more tightly coupled inter-firm relationship [3]. Especially, Clemons, et al. [13] argue that IT infrastructure leads firms to have less vertical relationships and move to long-term relationships with smaller number of partners by lowering coordination costs. This tightly coupled IT-mediated inter-firm relationship is called as electronic cooperation [4] and firms explicitly exploits IT infrastructure capabilities to establish this type of relationship.

In this study, we are concerned with electronic cooperation achieved through the implementation and use of IT infrastructure capability in inter-firm context. Key research questions that motivate our work are: (1) to identify the dimensions of IT infrastructure capability to be utilized for inter-firm relationship and (2) to examine the impacts of the dimensions on electronic cooperation. To answer the questions, we propose and test a model drawing from IT infrastructure framework, transaction costs theory, and information processing theory.

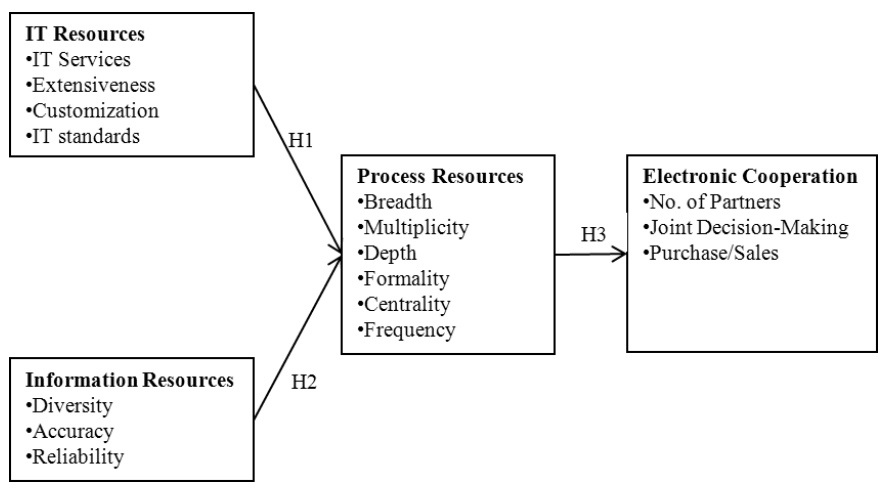

Fig. 1. Research Model

\section{RESEARCH MODEL AND HYPOTHESES}

A suitable research mode is developed to show how the three dimensions are interrelated and the impact of process resources on electronic cooperation. As shown in Figure 1, the research model and the associated hypotheses are elaborated. 


\section{A. IT Resources}

IT resources represent the provision of shared IT services on which inter-firm IT applications are implemented and used [8]. The shared IT services includes an integrated telecommunication network, task/team support technologies (e.g., groupware and discussion databases), information-rich media, and united customer database. In EDI-based ordering relationship, for example, manufacturers and suppliers utilize network protocol such as TCP/IP and XML and transaction sets for document exchange, and IT resource standards for processing transaction data.

Firms implement and use IT resources to significantly improve electronic interactions, such as electronic hubs and web services, with their trading partners [17].IT resources determine the nature and scope of technological integration between partners, which in turn, influences the degree of process integration of their relationships. For example, proprietary IT resources are likely to offer more customized IT functionality specific to existing inter-firm relationship. When IT resources provide extensive and customized IT services shared, firms in a relationship tend to build relation-specific process resources of IT infrastructure, which are forming relation-specific electronic interactions [5]. The formation of relation-specific linkages also enables firms in a relationship to exploit the IT infrastructure's benefits fully. The extensive and customized IT resources tend to make partnering firms build relation-specific workflows, processes, and human skills. Moreover, they delineate the "reach" of partnering firms, which is described as the locations and frequencies the partners in a relationship can reach. Thus, when more extensive and customized IT resources are shared, partnering firms are likely to establish multiple and intensive coordination structures which link their various functional areas with a variety of interaction channels. Therefore, this leads to our first hypothesis.

Hypothesis 1: Customized and extensive IT resources of inter-organizational IT infrastructure are positively associated with multiple and intensive process resources of interorganizational IT infrastructure.

\section{B. Information Resources}

The support of information resources to diverse information and high-quality information exchange lead firms in a relationship to reduce or eliminate information distortion and allow them to form effective coordination structures for facilitating many business coordination activities such as forecasting, synchronizing production and delivery, and making inventory-related decisions [11]. The extent to which information resources can support to diverse information such as operational, tactical, and strategic information between firms is considered as a component of process resource of IT infrastructure capability. Therefore, such information resources reduce the extent of conflicts between firms and improve free and frequent communications with minimum obstacles and consequently, facilitate to establish multiple and intensive interactions structures between partnering firms. Thus, we propose the following hypothesis.

Hypothesis 2: Diverse and high-quality information resources of inter-organizational IT infrastructure are positively associated with multiple and intensive process resources of interorganizational IT infrastructure.

\section{Process Resources}

Organization studies and information processing perspective sees coordination structure as a central factor in understanding the development cooperative inter-firm relationship and as direct correspondence to the nature of electronic cooperation [10]. The multiple and intensive coordination structures formed by process resources of IT infrastructure help firms to execute structured and unstructured inter-firm business processes which in turn, produce business-process specificity and domain-knowledge specificity. These relation-specific processes and knowledge have little use outside the relationship and hold firms together into tightly coupled relationship. Moreover, multiple and intensive and coordination structures provide firms in a relationship suitable information processing capabilities to reduce equivocality and uncertainty, which are considered as a critical factor to cooperative relationship[16]. This leads our final hypothesis.

Hypothesis 3: Multiple and intensive process resources of inter-organizational IT infrastructure are positively associated with higher electronic cooperation between partnering firms.

\section{RESEARCH DESIGN}

We collected data through a self-administered questionnaire from IS managers in manufacturing and retailing industries. The research instrument packages contained a cover letter, questionnaire, and postage-paid reply envelopes were mailed to 926 IS/IT managers. E-mails inviting participation in this study were also sent. Of the 926 questionnaires mailed, 13 questionnaires were not delivered and 10 of received mails were discarded since their responses were incomplete or indicated that their firm did not use IT infrastructure with their partnering firms for transactions. As a result, our survey yielded 96 usable responses out of 913 remained and a response rate was $10.5 \%$.The response rate of 10.5 percent is acceptable with the survey studies of this nature.

The target subjects of data collection were IS managers in manufacturing and retailing industries. These industries have used more IT for business transactions for more than decades and have noticed the importance of closer relationships with their partners. Due to this, the choice of manufacturing and retail industries as the target population is becoming more common in the EDI, inter-organizational information systems (IOS), and IT infrastructure studies (e.g., [4].The unit of analysis for this study is a dyad, dyadic relationship between several large buyers and their respective suppliers. In order to obtain analyzable measures, data should be collected from the two firms in a dyad and combined their scores to obtain an overall measure. However, the 
dyad-informant method is empirically both risky and can be impractical. In order to solve this problem, this study focuses the view of buyers and suppliers independently. In other words, it measures one firm's assessment on the inter-organizational IT infrastructure capabilities and the same firm's evaluation on electronic cooperation.

\section{DATA ANALYSIS AND RESULTS}

A primary means of statistical analyses was structural equation modeling (SEM) using AMOS. AMOS is covariancebased SEM tool. Covariance-based SEM tests the a priori specified model against population estimates derived from the sample. Covariance-based techniques also emphasize the overall fit of the entire observed covariance matrix with the hypothesized covariance model. Because of deductive nature of present research, the SEM technique is employed.

\section{A. Analysis of the Instrument}

TABLE I. NDICATOR ESTIMATES AND RELIABILITY STATISTICS OF CONSTRUCTS

\begin{tabular}{|l|l|l|l|l|}
\hline Constructs & Parameters & $\begin{array}{l}\text { ML } \\
\text { Estimate }\end{array}$ & $\begin{array}{l}\text { t- } \\
\text { Value** }\end{array}$ & Reliability \\
\hline $\begin{array}{l}\text { IT Resources } \\
\text { (ITR) }\end{array}$ & ITR1 & 0.60 & & \\
& ITR2 & 0.62 & 4.88 & \\
& ITR3 & 0.86 & 5.92 & 0.80 \\
& ITR4 & 0.75 & 5.56 & \\
\hline Information & IR1 & 0.43 & & \\
Resources & IR2 & 0.76 & 4.00 & 0.75 \\
(IR) & IR3 & 0.88 & 4.14 & \\
\hline Process & PR1 & 0.69 & & \\
Resources & PR2 & 0.64 & 5.73 & \\
(PR) & PR3 & 0.57 & 5.16 & 0.83 \\
& PR4 & 0.57 & 5.18 & \\
& PR5 & 0.81 & 7.17 & \\
& PR6 & 0.70 & 6.22 & \\
\hline Electronic & EC1 & 0.96 & & \multirow{2}{*|}{} \\
Cooperation & EC2 & 0.94 & 18.75 & 0.95 \\
(EC) & EC3 & 0.87 & 14.78 & \\
\hline
\end{tabular}

The first item of indicator estimate in each construct is fixed at 1.00 and does not have a t-value.** All t-values are significant at $\mathrm{p}<0.01$.

To analyze our instrument, we performed tests on construct validity and reliability. Confirmatory factor analysis was conducted to examine the validity of the constructs. Reliability (internal consistency) is an indication of measurement accuracy, that is, the extent to which instrument produces consistent or error-free results. Reliabilities of the four constructs (i.e., IT resources, information resources, process resources, and electronic cooperation) ranged from 0.75 to 0.95 , hence internal consistency appeared to be high, as shown in Table 1. Factor loadings were all positive, significant, and above the cutoff value of 0.4 [20]. Construct validity is the extent to which an operationalization measures the concepts that it supposed to measure. Two types of construct validity were measured. Convergent validity was verified through the t-statistic for each ML estimate. Table 1 indicates that all 16 indicators contain no values significantly different from zero, supporting the adequacy of the constructs. By comparing construct variance extracted with correlations among constructs, discriminant validity was found to be acceptable as well.

\section{B. Analysis of Hypothesized Research Model}

The SEM methodology checks the fit between the variancecovariance matrix observed in the sample data and that implied by the research model. This fit is expressed using measures of goodness of fit. Because there is no consensus on a single or a set of measures to assess fit, studies report several measures. As most studies in IS area did, we report observed $\chi 2$, goodness-offit (GFI), adjusted goodness-of-fit (AGFI), comparative-fit-index (CFI), and the root mean square error of approximation (RMSEA). Values higher than 0.90 for GFI and CFI indicate a good fit[20], while AGFI values higher than 0.80 suggest a good fit of the hypothesized model[18]. For RMSEA, a value less than 0.1 is considered a good fit, and a value less than 0.05 is considered a very good fit of the data to the research model.

The hypothesized research model assumes IT resources (ITR) and information resources (IR) influence process resources (PR) which in turn, has impact on electronic cooperation (EC). Figure 2 presents a graphical representation of the model with path coefficients for each construct. The path coefficients and fit indices for the model are listed in Table 2 . The observed $\chi 2$ for the hypothesized model is $310.75(\mathrm{df}=100, \mathrm{p}=0.000)$. The GFI, AGFI, CFI, and RMSEA are 0.92, 0.81, 0.90 and 0.09 respectively. Adjusting for degrees of freedom, the normed value of $\chi 2$ is 3.11. All goodness-of-fit indices including the normed value of $\chi 2$ indicate that the hypothesized model is a good-fitting model.

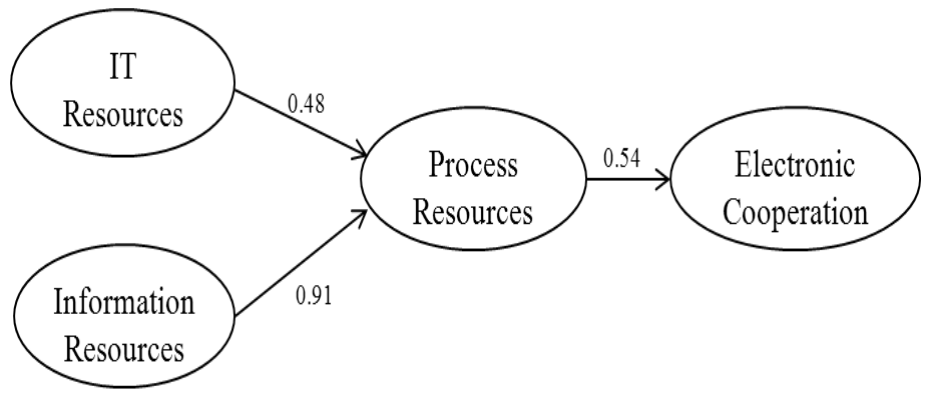

Fig. 2. Path analyze 
TABLE II. PATH AND FIT INDICES FOR HYPOTHESIZED MODEL

\begin{tabular}{|l|l|l|}
\hline Path & Coefficient $(\beta)$ & $\mathrm{t}$-Value \\
\hline $\begin{array}{l}\text { IT Resources } \\
\rightarrow>\text { Process Resources }\end{array}$ & 0.48 & $3.40 * *$ \\
\hline $\begin{array}{l}\text { Information Resources } \\
\rightarrow \text { Process Resources }\end{array}$ & 0.91 & $3.45^{* *}$ \\
\hline $\begin{array}{l}\text { Process Resources } \\
\rightarrow \text { Electronic Cooperation }\end{array}$ & 0.54 & $2.88^{*}$ \\
\hline $\begin{array}{l}\text { Measures of Model Fit } \\
\chi^{2}=310.75(\mathrm{df}=100 ; \mathrm{p}=0.000) ; \\
\text { GFI }=0.92 ; \text { AGFI }=0.81 ; \mathrm{CFI}=0.90 ; \text { RMSEA }=0.09\end{array}$ \\
* indicates $\mathrm{p}<0.05 ; * *$ indicates $\mathrm{p}<0.01$
\end{tabular}

As shown in Table 2 and Figure 2, most path coefficients are of high magnitude and exhibit high t-values. These values range from 0.48 to 0.91 . The $\mathrm{t}$-values indicates that all path coefficients are significant at $\mathrm{p}=0.01$ or 0.05 . Furthermore, the coefficient of determination for structural equations is 0.95 for the model, indicating that $95 \%$ of variance of electronic cooperation is explained by indicators. Therefore, $\mathrm{H} 1, \mathrm{H} 2$, and $\mathrm{H} 3$ were supported.

Cohen[14] provides recommendations for the interpretation of path coefficient $(\beta)$ in social science. Standardized path coefficients with absolute values less than 0.10 may indicate a "small" effect; values around 0.30 a "medium" one; and "large" effects may be suggested by coefficients with absolute values of 0.50 or more. Based on his recommendation, all constructs in the model have the values above 0.30 and their direction is positive. IT resources and information resources have moderate and strong association with process resources, respectively $(\beta=0.48$ and 0.91). Process resources also have strong association with electronic cooperation $(\beta=0.54)$.

\section{CONCLUSION}

This paper empirically tests the relationships among the three dimensions of IT infrastructure capability and the impacts of process resources on electronic cooperation. Our analysis provides better understanding about the roles of IT infrastructure on inter-firm relationships in two ways. First, this study extends the scope of IT infrastructure dimension. Previous researches have considered IT infrastructure as a single dimension (i.e., shared IT functionality) and investigated how the single dimension is related to inter-firm relationship. This study extends the dimensions to three: IT resources, information resources, and process resources. It confirms researchers' multi-dimensional view of IT infrastructure. Bensaou and Venkatraman[5] models three IT infrastructure dimensions to fulfill the needs for interfirm information processing capabilities.

Second, this study investigates the interdependencies among the dimensions of IT infrastructure capability. Our findings show the order of precedence among the dimensions. The strong path of IT and information resources $\rightarrow$ process resources $\rightarrow$ electronic cooperation is our potentially most influential finding. Instead the three dimensions individually have direct relationship to electronic cooperation, IT and information resources together influence processes resources so that firms can establish multiple and intensive coordination structures with their partners and the resulting coordination structures make them closer inter-firm relationships. As IT infrastructure provides extensively shared IT functionalities and customized IT platform and increases diverse and richer information exchanged, the equivocality and uncertainty accompanied to inadequate coordination structures formed in inter-firm relationships are reduced [16] and the opportunity for taking cooperative and coordinated actions in the relationships is also enhanced. This finding indicates that cooperative inter-firm relationship will be better achieved by supporting a variety of interaction channels for aligning business processes, communicating business strategies, building trust, and sharing information between partners. Our findings also confirm the association between structural governance and cooperative relationships stated in the studies in information systems, organization, and marketing areas [10].

\section{REFERENCES}

[1] R.Angeles, and R.,Nath, "Electronic Supply Chain Partnership: Reconsidering Relationship Attributes in Customer-Supplier Dyads", Information Resources Management Journal, vol. 16, no. 3, pp. 59-84, 2003

[2] N.Argyres, "The Impact of Information Technology on Coordination: Evidence from B-2 'Stealth' Bomber”, Organization Science, vol. 10, no. 2, pp. 162-180, 1999.

[3] A.Basu, and A.Kumar, "Research Commentary: Workflow Management Issues in e-Business", Information Systems Research, vol. 13, no. 1, pp. 1$14,2002$.

[4] M.Bensaou, "Interorganizational Cooperation: The Role of Information Technology: An Empirical Comparison of U.S. and Japanese Supplier Relations", Information Systems Research, vol. 8, no. 2, pp. 107-124, 1997

[5] M. Bensaou, and N. Venkatraman, "Configurations of Interorganizational Relationships: A Comparison between U.S. and Japanese Automakers", Management Science, vol. 41, no. 9, pp. 1471-1492, 1995

[6] P.A.Bernstein, and L.M.Hass, "Information Integration in the Enterprise", Communications of the ACM, vol. 51, no. 9, pp. 72-79, 2008.

[7] A.Bharadwaj, "A Resource-Based Perspective on Information Technology Capabilities and Firm Performance: An Empirical Investigation", MIS Quarterly, vol. 24, no. 1, pp. 169-196, 2000.

[8] M.Broadbent, P.Weill, and D. St. Clair, "The Implications of Information Technology Infrastructure for Business Process Redesign”, MIS Quarterly, vol. 23, no. 2, pp. 159-182, 1999.

[9] A. Bush, A. Tiwana, and A. Rai, "Complementarities between Product Design Modularity and IT Infrastructure Flexibility in IT-Enabled Supply Chains", IEEE Transactions on Engineering Management, vol. 57, no. 2, pp. $240-254,2010$.

[10] V.Choudhury, "Strategic Choices in the Development of Interorganizational Information Systems", Information Systems Research, vol. 8, no. 1, pp. 124, 1997.

[11] W.Y.Christina, K.L.Wong, and T.Cheng, "Value of Information Integration to Supply Chain Management: Role of Internal and External Contingencies", Journal of Management Information Systems, vol. 28, no. 3, pp. 161-199, 2011.

[12] T.H.Clark, and D.B.Stoddard, "Interorganizational Business Process Redesign: Merging Technological and Process Innovation", Journal of Management Information Systems, vol. 13, no. 2, pp. 9-28, 1996.

[13] E. K.Clemons, S. P. Reddi, and M.Row, "The Impact of Information Technology on the Organization of Economic Activity: The "Move to the 
Middle' Hypothesis", Journal of Management Information Systems, vol. 19, no. 2, pp. 9-35, 1993.

[14] J. Cohen, Statistical Power Analysis for the Behavioral Sciences, Academic Press, New York, 1988.

[15] CSC Index, Building the New Information Infrastructure,CSC Index, Boston, Mass., 1992.

[16] R.L.Daft, and R. H.Lengal, "Organizational Information Requirements, Media Richness and Structural Design”, Management Science,vol. 32, pp. 554-571, 1986.
[17] E. D.Daniel, and A.White, "The Future of Inter-Organisational System Linkages: Findings of an International Delphi Study", European Journal of Information Systems, vol. 14, no. 2, pp. 188-203, 2005.

[18] S.Devaraji, M.Fan, and R.Kohli, "Antecedents of B2C Channel Satisfaction and Preference: Validating e-Commerce Metrics”, Information Systems Research, vol. 13, no. 3, pp. 316-333, 2002.

[19] P.Evans, and T.Wurster, "A Strategy and the New Economics of Information”, Harvard Business Review, pp. 71-82, 1997.

[20] D.Gefen, D.Straub, and M.Boudreau, "Structural Equation Modeling and Regression: Guidelines for Research Practice", Communications of AIS, vol. 4 , no. 7 , pp. 1-76, 2000. 